\title{
Teaching research to undergraduate engineers
}

\section{George Nowak, Barry Shoop, Glen Dudevoir}

George A. Nowak, Barry L. Shoop, Glen Dudevoir, "Teaching research to undergraduate engineers," Proc. SPIE 9663, Eighth International Topical Meeting on Education and Training in Optics and Photonics, 96630 U (6 October 2003); doi: 10.1117/12.2208449

SPIE Event: Eighth International Topical Meeting on Education and Training in Optics and Photonics, 2003, Tucson, Arizona, United States 


\title{
Teaching research to undergraduate engineers
}

\author{
George A. Nowak, Barry L. Shoop, Glen Dudevoir \\ Department of Electrical Engineering and Computer Science \\ United States Military Academy, Bldg. 601, West Point, New York 10996 \\ george.nowak@usma.edu,Tel.854-938-5558,Fax 845-938-5956
}

\begin{abstract}
A method for introducing undergraduates to graduate-level research in electrical engineering is presented. The three-phase, year-long program involves extension of analytical theory from core and elective courses to a specialized research problem, construction and validation of numerical simulation techniques, and conduct of the experiment, with emphasis on analysis of results.

(C)2003 Optical Society of America

OCIS codes: (000.2060) Education; (000.2170) Equipment and techniques
\end{abstract}

\section{Introduction}

Typical undergraduate senior design project experiences do not equip students with skills necessary for pursuing graduate level research in engineering. We have constructed a year-long advanced individual study course to introduce high performing senior undergraduates to graduate-level research using a three-phase approach. The students review supporting theory from their coursework, and extend it to develop a simplified model for their research experiment. They then develop a numerical simulation program to refine the model to more-closely approximate the experiment, and validate the simulation program by reconciling it with their analytical solution. Finally, the students set up an experiment, take data, and reconcile results with predictions of simulations. In the last two years, student teams have successfully completed several sophisticated projects, including a high repetition rate, mode-locked fiber laser, and spectral continuum generation using photonic crystal fiber.

\section{Analytical Extension Phase}

Research topics are proposed to selected groups of 2-3 students at the outset of the course, based on electives that the students have completed, faculty areas of expertise, and probability of successful completion of the three phases of the program within a year. The proposed projects are in mature, but active, research areas with multiple recent theoretical and experimental publications. During the initial month of the program, an instructor customizes and extends concepts that the students have covered in courses to help them develop a reasonable analytical model for the research project. The intent of the bi-weekly seminars conducted during that month is to lead the students to a level such that they can understand the journal publications in the research area, and are able to construct a mathematical model for their research project. The conclusion of the phase is a simplified model for the experiment that the students can solve in closed form, and will serve as a validation benchmark for the numerical simulation. During the initial phase, the students are also led through experimental design, including familiarization with important specifications of components and instruments that will be used in the experiment.

\section{Simulation Phase}

Although students are exposed to various simulation tools, including MathCad and MATLAB, this phase of the program requires them to apply more sophisticated programming techniques to construct a numerical simulation that will reasonably predict experimental results. The emphasis is on validating the simulation program, and insuring that adequate variable parameters are included in the program to facilitate matching with experimental parameters.

\section{Experimental Phase}

In the final phase, student teams set up the experiment, and take data. The course emphasizes the proper use of a laboratory notebook to record details of experimental procedures and observations. Following the data acquisition, the team compares results with theoretical predictions, and reconciles discrepancies using error analysis procedures. The final requirement in the course is to write a journal-style paper to summarize findings. 